\title{
A quantitative approach to syndetic transitivity and topological ergodicity
}

\author{
Yu Zhao ${ }^{a}$, Risong Lij ${ }^{a, *}$, Tianxiu Lu ${ }^{b, c, d}$, Ru Jiang ${ }^{a}$, Hongqing Wang ${ }^{a}$, Haihua Liang ${ }^{a}$ \\ a School of Mathematic and Computer Science, Guangdong Ocean University, Zhanjiang, Guangdong, 524025, People's Republic of \\ China. \\ ${ }^{b}$ Department of Mathematics, Sichuan University of Science and Engineering, Zigong, Sichuan, 643000, People's Republic of China. \\ ${ }^{c}$ Artificial Intelligence Key Laboratory of Sichuan Province, Zigong, Sichuan, 643000, People's Republic of China. \\ ${ }^{d}$ Bridge Non-destruction Detecting and Engineering Computing Key Laboratory of Sichuan Province, Zigong, Sichuan, 643000, \\ People's Republic of China.
}

Communicated by A. Atangana

\begin{abstract}
In this paper, we give new quantitative characteristics of degrees of syndetical transitivity and topological ergodicity for a given discrete dynamical system, which are nonnegative real numbers and are not more than 1. For selfmaps of many compact metric spaces it is proved that a given selfmap is syndetically transitive if and only if its degree of syndetical transitivity is 1 , and that it is topologically ergodic if and only if its degree of topological ergodicity is one. Moreover, there exists a selfmap of $[0,1]$ having all degrees positive. (C) 2017 All rights reserved.
\end{abstract}

Keywords: Sensitivity, syndetically sensitive, ergodically sensitive, multi-sensitive, cofinitely sensitive, Furstenberg families. 2010 MSC: 37B10, 37C20, 37C50.

\section{Introduction}

In fact, a given dynamical system is a fixed pair $(A, t)$, where $t$ is a continuous selfmap of a topological space A. Various definitions of the behaviours of these systems have been given, such as topological transitivity, syndetical transitivity, topological ergodicity, topologically weak mixing, topological mixing, chaos and so on (see [1-22]). For many different definitions of chaos, topological transitivity is one of the ingredients. It is well-known that the classical definitions of chaos are usually qualitative. That is, by these definitions, one can only know that a given dynamical system is either chaotic or not. But by using these different definitions one can not know how large the chaos is. However, it is well-known that the topological entropy defined in [1] is the most important quantitative tool of chaoticity (also see [14]).

In [12], the authors gave degrees of transitivity, weak mixing and strong mixing that are several new quantitative characteristics which are nonnegative real numbers and are not more than 1 for given dynamical systems. Moreover, for selfmaps of many compact metric spaces they proved that a given

\footnotetext{
${ }^{*}$ Corresponding author

Email addresses: datom@189.cn (Yu Zhao), gdoulrs@163.com (Risong Li), lubeeltx@163.com (Tianxiu Lu), jiru1995@163.com (Ru Jiang), wanghq3333@126.com (Hongqing Wang), lhhlucy@126.com (Haihua Liang)

doi:10.22436/jnsa.010.09.10
} 
selfmap is topologically transitive (resp. weak mixing or strong mixing) if and only if its degree of transitivity (resp. weak mixing or strong mixing) is 1 . Furthermore, there is a simple selfmap on $[0,1]$ with all degrees positive. Snydetical transitivity and topological ergodicity are qualitative characteristics of a given selfmap of a metric space, but inspired by [12] one can find a way how to measure to which extent snydetical transitivity or topological ergodicity is present in the dynamics of a given selfmap such that the result is a nonnegative real number and is not more than 1, and that the value 1 means that the system satisfies the definition of syndetical transitivity or topological ergodicity. Also, it is well-known that the syndetical transitivity (resp. topological ergodicity) is an important and stronger transitivity. This property may happen in a variety of applications. So, it is not surprising that in the physics and other sciences there was a lot of effort to quantify syndetical transitivity (resp. topological ergodicity) and compare the complexity of a given selfmap in such a tool. In this paper, inspired by idea of [12] we are going to rigorously define degrees of syndetical transitivity and topological ergodicity of given selfmaps. In particular, for given selfmaps of many compact metric spaces it is proven that a given selfmap is syndetically transitive if and only if its degree of syndetical transitivity is 1 , and that it is topologically ergodic if and only if its degree of topological ergodicity is one.

In Section 2, some basic concepts and notation are recalled, and the definitions of the degrees of syndetical transitivity and topological ergodicity are given. Our results are obtained in Section 3 . We conclude this paper with some comments in the last section.

\section{Preliminaries}

A dynamical system is a pair $(A, t)$ such that $A$ is a compact metric space, and that $t$ is a continuous selfmap of $A$.

A system $(A, t)$ (or a map $t: A \rightarrow A$ ) is topologically transitive if and only if for any two nonempty open subsets $X, Y \subset A$ we can find an integer $l \geqslant 0$ with $t^{l}(X) \cap Y \neq \emptyset$.

A system $(A, t)$ (or a map $t: A \rightarrow A$ ) is (topologically) weakly mixing if and only if $t \times t$ is topologically transitive.

A system $(A, t)$ (or a map $t: A \rightarrow A$ ) is topologically mixing (or strongly mixing) if and only if for any two nonempty open subsets $X, Y \subset A$ one can find an integer $l_{0} \geqslant 0$ with $t^{l}(X) \cap X \neq \emptyset$ for any $l \geqslant l_{0}$.

Write $Z^{+}=\{0,1,2, \cdots\}$. A subset $E \subset Z^{+}$is thick, if $E$ can contain arbitrarily large blocks of consecutive numbers. A subset $E \subset Z^{+}$is syndetic, if $Z^{+} \backslash E$ is not thick.

A dynamical system $(A, t)$ or a map $t: A \rightarrow A$ is syndetically transitive (see $[4,11,16,18])$, if and only if the set $N_{t}(X, Y)$ is syndetic for any two nonempty open subsets $X, Y \subset A$, where

$$
\mathrm{N}_{\mathrm{t}}(X, Y)=\left\{l \in Z^{+}: \mathrm{t}^{\mathrm{l}}(X) \cap \mathrm{Y} \neq \emptyset\right\}
$$

A dynamical system $(A, t)$ or a map $t: A \rightarrow A$ is topologically ergodic, if and only if the set $N_{t}(X, Y)$ has positive upper density for any two nonempty open subsets $X, Y \subset A$, that is,

$$
\limsup _{l \rightarrow \infty} \frac{1}{l}\left|N_{f}(X, Y) \cap\{0,1, \cdots, l-1\}\right|>0,
$$

where $|\mathrm{D}|$ denotes the cardinality of $\mathrm{D}$ (see $[3,4,15,19])$.

Clearly, by the definitions, syndetical transitivity implies topological ergodicity, and topological ergodicity implies transitivity. Moreover, topological mixing implies syndetical transitivity, and it is well-known that the converse implications is not necessarily true.

Let $\mathrm{t}$ be a continuous selfmap of a compact metric space $A$. For any given finite cover $\mathscr{D}$ of $\mathrm{A}$ and any given integer $\mathrm{m} \geqslant 1$, the cell $\mathrm{C} \times \mathrm{C}^{\prime} \in \mathscr{D} \times \mathscr{D}$ is $\mathrm{T}^{\mathrm{m}}$-admissible if $\mathrm{t}^{\mathrm{m}}(\mathrm{C}) \cap \mathrm{C}^{\prime} \neq \emptyset$ (see [12]). Let $\mathscr{A}\left(\mathrm{t}^{\mathrm{m}}, \mathscr{D}\right)$ be the set of all $\mathrm{t}^{\mathrm{m}}$-admissible cells from $\mathscr{D} \times \mathscr{D}$. Moreover, we use $\mathscr{A}^{\mathrm{m} \geqslant 1}(\mathrm{t}, \mathscr{D}), \mathscr{A}^{\infty}(\mathrm{t}, \mathscr{D}), \mathscr{A}^{\operatorname{cofin}}(\mathrm{t}, \mathscr{D})$ to denote the set of all cells $\mathrm{C} \times \mathrm{C}^{\prime} \in \mathscr{D} \times \mathscr{D} \mathscr{D}$ which are $\mathrm{t}^{\mathrm{m}}$-admissible for at least one $\mathrm{m}>0$, for infinitely many m's, for all but finitely many m's, respectively. Therefore, one has that 


$$
\begin{aligned}
& \mathscr{A}^{\mathrm{m} \geqslant 1}(\mathrm{t}, \mathscr{D})=\bigcup_{\mathrm{m}=1}^{\infty} \mathscr{A}\left(\mathrm{t}^{\mathrm{m}}, \mathscr{D}\right), \\
& \mathscr{A}^{\infty}(\mathrm{t}, \mathscr{D})=\limsup _{\mathrm{m} \rightarrow \infty} \mathscr{A}\left(\mathrm{t}^{\mathrm{m}}, \mathscr{D}\right),
\end{aligned}
$$

and

$$
\mathscr{A}^{\text {cofin }}(\mathrm{t}, \mathscr{D})=\liminf _{\mathrm{m} \rightarrow \infty} \mathscr{A}\left(\mathrm{t}^{\mathrm{m}}, \mathscr{D}\right) .
$$

Let $\mathrm{B}$ denote the cardinality of a set B. Then, the ratio $\frac{\left|\mathscr{A}\left(\mathrm{t}^{\mathrm{m}}, \mathscr{D}\right)\right|}{|\mathscr{D}|^{2}}$ can show how large portion of cells from $\mathscr{D} \times \mathscr{D}$ is intersected by the graph of the map $\mathrm{t}^{\mathrm{m}}$. Obviously, this ratio is always a real number from $\left[\frac{1}{|\mathscr{D}|}, 1\right]$. Analogously for $\mathscr{A}\left(\mathrm{t}^{\mathrm{m}}, \mathscr{D}\right)$ replaced by $\mathscr{A}^{\mathrm{m} \geqslant 1}(\mathrm{t}, \mathscr{D})$ or $\mathscr{A}^{\infty}(\mathrm{t}, \mathscr{D})$. Moreover, the case of that $\mathscr{A}^{\text {cofin }}(\mathrm{t}, \mathscr{D})=\emptyset$ can be true.

For a given a finite cover $\mathscr{D}$ of $A,{ }^{*}$-degree of strong mixing, degree of strong mixing, degree of weak mixing, degree of transitivity and ${ }^{*}$-degree of transitivity of $\mathrm{t}$ with respect to this cover $\mathscr{D}$ are defined by $\mathrm{dsm}^{*}(\mathrm{t}, \mathscr{D})=\frac{\left|\mathscr{A}^{\text {cofin }}(\mathrm{t}, \mathscr{D})\right|}{|\mathscr{D}|^{2}}, \operatorname{dsm}(\mathrm{t}, \mathscr{D})=\liminf _{\mathrm{m} \rightarrow \infty} \frac{\left|\mathscr{A}^{\text {cofin }}\left(\mathrm{t}^{\mathrm{m}}, \mathscr{D}\right)\right|}{|\mathscr{D}|^{2}}, \operatorname{dwm}(\mathrm{t}, \mathscr{D})=\limsup _{\mathrm{m} \rightarrow \infty} \frac{\left|\mathscr{A}^{\operatorname{cofin}}\left(\mathrm{t}^{\mathrm{m}}, \mathscr{D}\right)\right|}{|\mathscr{D}|^{2}}$, $\mathrm{dt}(\mathrm{t}, \mathscr{D})=\frac{\left|\mathscr{A}^{\infty}(\mathrm{t}, \mathscr{D})\right|}{|\mathscr{D}|^{2}}$ and $\mathrm{dt}^{*}(\mathrm{t}, \mathscr{D})=\frac{|\mathscr{A} \geqslant 1(\mathrm{t}, \mathscr{D})|}{|\mathscr{D}|^{2}}$, respectively (see [12]). Inspired by the above definitions we introduce two new quantitative characteristics of a given dynamical system $(A, t)$.

Definition 2.1. For a given finite cover $\mathscr{A}$ of $A$ and a given dynamical system $(A, t)$, we give degree of syndetical transitivity and degree of topological ergodicivity of $t$ with respect to this cover $\mathscr{D}$ respectively, by $\operatorname{dst}(\mathrm{t}, \mathscr{D})=\frac{\left|\mathscr{A}^{\mathrm{syn}}(\mathrm{t}, \mathscr{D})\right|}{|\mathscr{D}|^{2}}$ and dte $(\mathrm{t}, \mathscr{D})=\frac{\left|\mathscr{A}^{\mathrm{erg}}(\mathrm{t}, \mathscr{D})\right|}{|\mathscr{C}|^{2}}$, where $\mathscr{A}^{\mathrm{syn}}(\mathrm{t}, \mathscr{D})=\left\{\mathrm{C} \times \mathrm{C}^{\prime} \in \mathscr{D} \times \mathscr{D}\right.$ : there exists a syndetic set $\mathrm{S} \subset \mathrm{Z}^{+}$such that $\mathrm{C} \times \mathrm{C}^{\prime}$ is $\mathrm{t}^{\mathrm{m}}$-admissible for any $\left.\mathrm{m} \in \mathrm{S}\right\}$ and $\mathscr{A}^{\mathrm{erg}}(\mathrm{t}, \mathscr{D})=\left\{\mathrm{C} \times \mathrm{C}^{\prime} \in \mathscr{D} \times \mathscr{D}\right.$ : there exists a set $S \subset Z^{+}$with positive upper density such that $C \times C^{\prime}$ is $t^{m}$-admissible for any $m \in S$.

It is clear that for any given dynamical system $(A, t)$ and any given cover $\mathscr{C}$, one has that

$$
0 \leqslant \operatorname{dsm}^{*}(t, \mathscr{D}) \leqslant \operatorname{dsm}(t, \mathscr{D}) \leqslant \operatorname{dst}(t, \mathscr{D}) \leqslant \operatorname{dte}(t, \mathscr{D}) \leqslant 1,
$$

and

$$
0 \leqslant \mathrm{dsm}(\mathrm{t}, \mathscr{D}) \leqslant \mathrm{dwm}(\mathrm{t}, \mathscr{D}) \leqslant \mathrm{dt}(\mathrm{t}, \mathscr{D}) \leqslant \mathrm{dt} \mathrm{t}^{*}(\mathrm{t}, \mathscr{D}) \leqslant 1
$$

\section{Main results}

Theorem 3.1. Let $(\mathrm{A}, \mathrm{t})$ be a given dynamical system. Then the following hold.

(a) $\mathrm{t}$ is syndetically transitive if and only if $\mathrm{dst}(\mathrm{t}, \mathscr{D})=1$ for any finite open cover $\mathscr{C}$.

(b) $\mathrm{t}$ is topologically ergodic if and only if $\mathrm{dte}(\mathrm{t}, \mathscr{D})=1$ for any finite open cover $\mathscr{D}$.

Proof.

(a) Suppose that $t$ is syndetically transitive and $\mathscr{D}$ is a given finite open cover of $A$. Then, by the definition, $\mathrm{N}_{\mathrm{t}}\left(\mathrm{C}, \mathrm{C}^{\prime}\right)$ is syndetic for any $\mathrm{C}, \mathrm{C}^{\prime} \in \mathscr{D}$. This means $\mathrm{C} \times \mathrm{C}^{\prime} \in \mathscr{A}^{\mathrm{syn}}(\mathrm{t}, \mathscr{D})$. So, $\operatorname{dst}(\mathrm{t}, \mathscr{D})=1$ for any given finite open cover $\mathscr{D}$.

Now we suppose that $\operatorname{dst}(\mathrm{t}, \mathscr{D})=1$ for any given finite open cover $\mathscr{D}$. Let $\mathrm{W}, \mathrm{Y} \subset \mathrm{A}$ be nonempty and open subsets. Choose any finite open cover $\mathscr{D}_{0}$ such that $\mathrm{W}, \mathrm{Y} \in \mathscr{D}_{0}$. Then $\mathrm{W} \times \mathrm{Y} \in \mathscr{A}^{\mathrm{syn}}\left(\mathrm{t}, \mathscr{D}_{0}\right)$, which implies that $N_{t}(W, Y)$ is syndetic. That is, $t$ is syndetically transitive.

(b) Suppose that $t$ is topologically ergodic and $\mathscr{D}$ is a given finite open cover of $A$. By the definition, $\mathrm{N}_{\mathrm{t}}\left(\mathrm{C}, \mathrm{C}^{\prime}\right)$ has positive upper density for any $\mathrm{C}, \mathrm{C}^{\prime} \in \mathscr{D}$. This means $\mathrm{C} \times \mathrm{C}^{\prime} \in \mathscr{A}^{\mathrm{erg}}(\mathrm{t}, \mathscr{D})$. So, $\operatorname{dte}(\mathrm{t}, \mathscr{D})=1$ for any given finite open cover $\mathscr{D}$ of $A$. 
Now, we suppose that dte $(t, \mathscr{D})=1$ for every finite open cover $\mathscr{D}$. Let $\mathrm{W}, \mathrm{Y} \subset \mathrm{A}$ be nonempty and open subsets. Choose any finite open cover $\mathscr{D}_{0}$ such that $W, Y \in \mathscr{D}_{0}$. Then $W \times Y \in \mathscr{A}^{\operatorname{erg}}\left(\mathrm{t}, \mathscr{D}_{0}\right)$, which implies that $N_{t}(W, Y)$ has positive upper density. That is, $t$ is topologically ergodic.

Thus, the entire proof is finished.

Let $A$ be a compact metric space with a metric s. For any $W \subset A$ and any $\alpha>0$, let $N(W, \alpha)$ be the smallest number of $\alpha$-balls (open balls with radius $\alpha$ ) needed to cover $W$, that is,

$$
N(W, \alpha)=\min \left\{l \in \mathbb{N}: W \subset \bigcup_{j=1}^{l} B\left(y_{j}, \alpha\right) \text { for some } y_{1}, y_{2}, \cdots, y_{l} \in X\right\},
$$

where $B(y, \alpha)$ is the $\alpha$-ball with center $y$ (see [12]). It is easily seen that $\lim _{\alpha \rightarrow 0^{+}} N(W, \alpha)=\infty$ if and only if $W$ is infinite.

A cover with no proper subcover is said to be minimal. A cover $\mathscr{D}$ of $A$ is said to be an $\alpha$-cover if any element of $\mathscr{D}$ is an $\alpha$-ball. An $\alpha$-cover of a compact metric space $A$ is economical (see [12]) if and only if its cardinality equals $N(A, \alpha)$, that is, if it is an $\alpha$-cover of $A$ with minimal cardinality. Let $\mathscr{E}(A, \alpha)$ be the set of all economical $\alpha$-covers of $A$ (see [12]).

For a continuous selfmap $t$ of a compact metric space $A$, its ${ }^{*}$-degree of strong mixing, degree of strong mixing, degree of weak mixing, degree of transitivity and *-degree of transitivity (see [12]) are given, respectively, by

$$
\begin{aligned}
\operatorname{dsm}^{*}(t) & =\liminf _{\alpha \rightarrow 0^{+}} \min _{\mathscr{D} \in \mathscr{E}(A, \alpha)} \operatorname{dsm}^{*}(t, \mathscr{D}), \\
\operatorname{dsm}(t) & =\liminf _{\alpha \rightarrow 0^{+}} \min _{\mathscr{D} \in \mathscr{E}(A, \alpha)} \operatorname{dsm}(t, \mathscr{D}), \\
\operatorname{dwm}(t) & =\liminf _{\alpha \rightarrow 0^{+}} \min _{\mathscr{D} \in \mathscr{E}(A, \alpha)} \operatorname{dwm}(t, \mathscr{D}), \\
\operatorname{dt}(t) & =\liminf _{\alpha \rightarrow 0^{+}} \min _{\mathscr{D} \in \mathscr{E}(A, \alpha)} \operatorname{dt}(t,),
\end{aligned}
$$

and

$$
d t^{*}(t)=\liminf _{\alpha \rightarrow 0^{+}} \min _{\mathscr{D} \in \mathscr{E}(A, \alpha)} d t^{*}(t, \mathscr{D}) .
$$

Inspired by these definitions, we give the following definitions.

Definition 3.2. For a continuous selfmap t of a compact metric space $A$, its degree of syndetical transitivity and degree of topological ergodicity are given, respectively, by

$$
\operatorname{dst}(t)=\liminf _{\alpha \rightarrow 0^{+}} \min _{\mathscr{D} \in \mathscr{E}(A, \alpha)} \operatorname{dst}(t, \mathscr{D}),
$$

and

$$
\operatorname{dte}(t)=\liminf _{\alpha \rightarrow 0^{+}} \min _{\mathscr{D} \in \mathscr{E}(A, \alpha)} \operatorname{dte}(t, \mathscr{D}) .
$$

From the definitions it follows that

$$
0 \leqslant \operatorname{dsm}^{*}(t) \leqslant \operatorname{dsm}(t) \leqslant \operatorname{dst}(t) \leqslant \operatorname{dte}(t) \leqslant 1,
$$

and

$$
0 \leqslant d s m(t) \leqslant d w m(t) \leqslant d t(t) \leqslant d t^{*}(t) \leqslant 1 .
$$

Further, for any $m \geqslant 1$ we have that $\operatorname{dsm}^{*}\left(t^{m}\right) \geqslant d s m^{*}(t), \operatorname{dsm}\left(t^{m}\right) \geqslant d s m(t), \operatorname{dwm}(t) \geqslant \operatorname{dwm}\left(t^{m}\right)$, $d t(t) \geqslant d t\left(t^{m}\right), d t^{*}(t) \geqslant d t^{*}\left(t^{m}\right), \operatorname{dst}(t) \geqslant d s t\left(t^{m}\right)$, and dte $(t) \geqslant \operatorname{dte}\left(t^{m}\right)$.

Theorem 3.3. Let $\mathrm{t}$ be a continuous selfmap of a compact metric space A. Then the following hold. 
(a) The syndetical transitivity of $\mathrm{t}$ implies $\operatorname{dst}(\mathrm{t})=1$.

(b) The topological ergodicity of $\mathrm{t}$ implies dte $(\mathrm{t})=1$.

Proof. By Theorem 3.1 and the definitions, Theorem 3.3 is true.

The following example shows that in general, the converse implications of Theorem 3.3 are not true.

Example 3.4 ([12]). Let $A=\mathrm{I}^{2} \cup([-1,0] \times\{0\})$ with Euclidean metric, where $\mathrm{I}=[0,1]$. Choose any transitive or topologically weakly mixing or topologically mixing or syndeticaly transitive or topologically ergodic continuous selfmap $q$ of $\mathrm{I}^{2}$ with $(0,0)$ as a fixed point and extend it to a continuous selfmap $t$ of A by putting $t(y, 0)=(0,0)$ for any $y \in[-1,0)$. Then $t$ is not transitive (or topologically weakly mixing or topologically mixing or syndetically transitive or topologically ergodic). Furthermore, it is well-known that the degrees of $t$ are the same as the degrees of $q$.

The above example shows that in order to give full characterization of transitivity, topologically weak mixing, topological mixing, syndetical transitivity and topological ergodicity of a given continuous selfmap on a compact metric space the above degrees, some additional "regularity" assumption on $A$ is required. This kind of assumption is said to be weak regularity (see [12]). For a given compact metric space $A$ and any given subset $B \subset A$, let

$$
\mu(B)=\liminf _{\alpha \rightarrow 0^{+}} \frac{N(B, \alpha)}{N(A, \alpha)} .
$$

Note that there are examples showing that this limit may be not exist (see [12]). A compact metric space $A$ is weakly regular, if and only if $\mu(W)>0$ for any nonempty open subset $W$ of $A$. A dynamical system $(A, t)$ or a continuous selfmap $t$ on a compact metric space is weakly regular, if and only if $A$ is weakly regular (see [12]). It is known from [12] that any compact manifold is weakly regular, and that the Cantor ternary set is weakly regular. However, it is well-known that the space A from Example 3.4 is not weakly regular, and that any infinite compact metric space with an isolated point is also not weakly regular.

Theorem 3.5. If $(A, t)$ is a given weakly regular dynamical system, then the following hold.

(a) the map $\mathrm{t}$ is syndetically transitive if and only if $\mathrm{dst}(\mathrm{t})=1$.

(b) the map $\mathrm{t}$ is topologically ergodic if and only if $\mathrm{dte}(\mathrm{t})=1$.

By Theorem 3.5 we know that for any given weakly regular dynamical system, the maximal value $(=1)$ of any of the above two degrees is a topological invariant. The following two lemmas which come from [12] are useful.

Lemma 3.6 ([12]). If $\mathscr{D}$ is a given economical $\alpha$-cover of $\mathrm{A}$ and $\mathrm{W} \subset \mathrm{A}$ is open, and if $\mathscr{D} \mathrm{W}=\{\mathrm{F} \in \mathscr{D}: \mathrm{D} \subset \mathrm{W}\}$, then $\mathrm{N}\left(\right.$ int $\left._{2 \alpha} W, \alpha\right) \leqslant\left|\mathscr{D}_{W}\right| \leqslant \mathrm{N}(\mathrm{W}, \alpha)$, where int ${ }_{2 \alpha} \mathrm{W}=\{\mathrm{y} \in \mathrm{W}: \mathrm{B}(\mathrm{y}, 2 \alpha) \subset \mathrm{W}\}$.

Lemma 3.7 ([12]). If $A$ is a given weakly regular and $W, T \subset A$ is nonempty and open, then there exist

$$
\alpha_{0}=\alpha_{W, T}>0 \text { and } \lambda=\lambda_{W, T},
$$

satisfying that for any continuous selfmap $\mathrm{t}$ of $\mathrm{A}$, any $\alpha \in\left(0, \alpha_{0}\right)$ and any integer $\mathrm{m}>0, \mathrm{t}^{\mathrm{m}}(\mathrm{W}) \cap \mathrm{T}=\emptyset$ implies $\frac{\left|\mathscr{A}\left(\mathrm{t}^{\mathrm{m}}, \mathscr{D}\right)\right|}{|\mathscr{D}|^{2}} \leqslant 1-\lambda$ for any $\mathscr{D} \in \mathscr{E}(A, \alpha)$.

Proof of Theorem 3.5.

(a) By Theorem 3.3 it is enough to prove that if $\operatorname{dst}(t)=1$ then $t$ is syndetically transitive. Suppose on the contrary that $t$ is not syndetically transitive. Then, by the definition there exist two open subsets $W, Y$ of A with

$$
W, Y \neq \emptyset,
$$

such that for any positive integer $L$ there is $m_{L}$ satisfying that $f^{j}(W) \cap Y=\emptyset$ for any integer

$$
j \in\left[m_{L}, m_{L}+L\right] .
$$

Let $\mathscr{D}_{W}$ and $\mathscr{D}_{Y}$ be defined as in Lemma 3.6. Choose $\alpha_{0}>0$ and $\lambda>0$ satisfying that both int $2 \alpha_{0} W$ 
and $\operatorname{int}_{2 \alpha_{0}} Y$ are nonempty, and that $N\left(\operatorname{int}_{2 \alpha} W, \alpha\right) \geqslant \sqrt{\lambda} N(A, \alpha)$ and $N\left(\operatorname{int}_{2 \alpha} Y, \alpha\right) \geqslant \sqrt{\lambda} N(A, \alpha)$ for any $0<\alpha<\alpha_{0}$. From the proof of [12, Lemma 11], we know that for any continuous selfmap $t$ on $A$, any $\alpha \in\left(0, \alpha_{0}\right)$ and any $\mathscr{D} \in \mathscr{E}(A, \alpha)$, if $\mathrm{t}^{j}(\mathrm{~W}) \cap \mathrm{Y}=\emptyset$ then $\mathscr{A}\left(\mathrm{t}^{j}, \mathscr{D}\right) \cap\left(\mathscr{D}_{W} \times \mathscr{D}_{Y}\right)=\emptyset$. This implies that $\frac{\left|\mathscr{A}\left(\mathrm{t}^{\mathrm{j},}, \mathscr{D}\right)\right|}{|\mathscr{D}|^{2}} \leqslant 1-\lambda$ for any $\mathscr{D} \in \mathscr{E}(A, \alpha)$, any integer $\mathrm{L}$, some integer $\mathrm{m}_{\mathrm{L}}$ and any integer $j \in\left[\mathrm{m}_{\mathrm{L}}, \mathrm{m}_{\mathrm{L}}+\mathrm{L}\right]$. So, by the definitions of $\operatorname{dst}(\mathrm{t}, \mathscr{D})$ and $\mathscr{A}^{\mathrm{syn}}(\mathrm{t}, \mathscr{D})$ we have $\operatorname{dst}(\mathrm{t}, \mathscr{D}) \frac{\left|\mathscr{A}\left(\mathrm{t}^{\mathrm{j}}, \mathscr{D}\right)\right|}{|\mathscr{D}|^{2}} \leqslant 1-\lambda$ for some $\mathrm{j}>0$. This is a contradiction.

(b) By Theorem 3.3 it is enough to prove that if $\mathrm{dte}(\mathrm{t})=1$, then $\mathrm{t}$ is topologically ergodic. Assume on the contrary that $t$ is not topologically ergodic. Then, by the definition there are two open subsets $W, Y$ of $A$ with $W, Y \neq \emptyset$ and $S \subset\{0,1,2, \cdots\}$ with $\limsup _{n \rightarrow \infty} \frac{|S \cap\{0,1, \cdots, n-1\}|}{n}=1$ such that $t^{j}(W) \cap Y=\emptyset$ for any integer $j \in S$. Let $\mathscr{D}_{W}$ and $\mathscr{D}_{Y}$ be defined as in Lemma 3.6. Choose $\alpha_{0}>0$ and $\lambda>0$ satisfying that both $\operatorname{int}_{2 \alpha_{0}} W$ and $\operatorname{int}_{2 \alpha_{0}} Y$ are nonempty, and that $N\left(\operatorname{int}_{2 \alpha} W, \alpha\right) \geqslant \sqrt{\lambda} N(A, \alpha)$ and $N\left(\operatorname{int}_{2 \alpha} Y, \alpha\right) \geqslant \sqrt{\lambda} N(A, \alpha)$ for any $0<\alpha<\alpha_{0}$. From the proof of [12, Lemma 11] we know that for any continuous selfmap $t$ on $A$, any $\alpha \in\left(0, \alpha_{0}\right)$ and any $\mathscr{D} \in \mathscr{E}(A, \alpha)$, if $\mathrm{t}^{\mathrm{j}}(\mathrm{W}) \cap \mathrm{Y}=\emptyset$ then $\mathscr{A}\left(\mathrm{t}^{j}, \mathscr{D}\right) \cap\left(\mathscr{D}_{W} \times \mathscr{D}_{Y}\right)=\emptyset$. This implies that $\frac{\left|\mathscr{A}\left(\mathrm{t}^{\mathrm{j}}, \mathscr{D}\right)\right|}{|\mathscr{D}|^{2}} \leqslant 1-\lambda$ for any $\mathscr{D} \in \mathscr{E}(A, \alpha)$ and any integer $j \in S$. So, by the definitions of dte $(\mathrm{t}, \mathscr{D})$ and $\mathscr{A}^{\operatorname{erg}}(\mathrm{t}, \mathscr{D})$ we have $\mathrm{dte}(\mathrm{t}, \mathscr{D}) \frac{\left|\mathscr{A}\left(\mathrm{t}^{\mathrm{j}}, \mathscr{D}\right)\right|}{|\mathscr{D}|^{2}} \leqslant 1-\lambda$ for some $\mathrm{j} \in \mathrm{S}$. It is a contradiction.

Thus, the entire proof is finished.

Remark 3.8. From [12, Proposition 12] and its proof, we know that there is a continuous selfmap $t$ on the unit interval $J=[0,1]$ which is of type 1 (even $t(y) \geqslant y$ for any $y \in J)$ and has all seven degrees positive.

\section{Conclusion}

In this paper we present new quantitative characteristics of degrees of syndetical transitivity and topological ergodicity for a discrete dynamical system, which are nonnegative real numbers and are not more than 1. For selfmaps of many compact metric spaces, we prove that a selfmap is syndetically transitive (resp. topologically ergodic) if and only if its degree of syndetical transitivity (resp. topological ergodicity) is one. Also, there is a selfmap of $[0,1]$ with all positive degrees of the above seven transitivity properties. Based on some conclusions obtained by Snoha and Špitalský in [12] and us in this paper, we will further discuss some applications of the quantitative characteristics of the above seven degrees in the future.

\section{Acknowledgement}

The authors are very grateful to the referees for their careful reading, comments, and suggestions, which help us improve this paper.

This research was supported by the Project of Enhancing School With Innovation of Guangdong Ocean University (Grant NO. GDOU2016050207), the Key Scientific and Technological Research Project of Science and Technology Department of Zhanjiang City (Grant 2010C3112005), the National Natural Science Foundation of China (11501391), the Opening Project of Artificial Intelligence Key Laboratory of Sichuan Province (2015RZJ01) and the Opening Project of Bridge Non-destruction Detecting and Engineering Computing Key Laboratory of Sichuan Province (2014QZJ02).

\section{References}

[1] R. L. Adler, A. G. Konheim, M. H. McAndrew, Topological entropy, Trans. Amer. Math. Soc., 114 (1965), 309-319. 1

[2] R. Li, A note on the three versions of distributional chaos, Commun. Nonlinear Sci. Numer. Simul., 16 (2011), 19931997.

[3] R. Li, A note on shadowing with chain transitivity, Commun. Nonlinear Sci. Numer. Simulat., 17 (2012), $2815-2823$. 2

[4] R. Li, A note on stronger forms of sensitivity for dynamical systems, Chaos Solitons Fractals, 45 (2012), 753-758.2 
[5] R. Li, The large deviations theorem and ergodic sensitivity, Commun. Nonlinear Sci. Numer. Simul., 18 (2013), $819-825$.

[6] R. Li, A note on decay of correlation implies chaos in the sense of Devaney, Appl. Math. Model., 39 (2015), 6705-6710.

[7] R. Li, A note on chaos and the shadowing property, Int. J. Gen. Syst., 45 (2016), 675-688.

[8] R. Li, Y. Shi, Stronger forms of sensitivity for measure-preserving maps and semiflows on probability spaces, Abstr. Appl. Anal., 2014 (2014), 10 pages.

[9] R. Li, H.-Q. Wang, Y. Zhao, Kato's chaos in duopoly games, Chaos Solitons Fractals, 84 (2016), 69-72.

[10] R. Li, X.-L. Zhou, A note on chaos in product maps, Turkish J. Math., 37 (2013), 665-675.

[11] T. K. S. Moothathu, Stronger forms of sensitivity for dynamical systems, Nonlinearity, 20 (2007), 2115-2126. 2

[12] L. Snoha, V. Špitalský, A quantitative approach to transitivity and mixing, Chaos Solitons Fractals, 40 (2009), $958-965$. $1,2,3,3.4,3,3,3.6,3.7,3,3.8,4$

[13] X.-X. Wu, Chaos of transformations induced onto the space of probability measures, Internat. J. Bifur. Chaos Appl. Sci. Engrg., 26 (2016), 12 pages.

[14] X.-X. Wu, A remark on topological sequence entropy, Internat. J. Bifur. Chaos Appl. Sci. Engrg., 27 (2017), 7 pages. 1

[15] X.-X. Wu, G.-R. Chen, Sensitivity and transitivity of fuzzified dynamical systems, Inform. Sci., 396 (2017), 14-23. 2

[16] X.-X. Wu, P. Oprocha, G.-R. Chen, On various definitions of shadowing with average error in tracing, Nonlinearity, 29 (2016), 1942-1972. 2

[17] X.-X. Wu, X. Wang, On the iteration properties of large deviations theorem, Internat. J. Bifur. Chaos Appl. Sci. Engrg., 2016 (2016), 6 pages.

[18] X.-X. Wu, J.-J. Wang, G.-R. Chen, $\mathscr{F}$-sensitivity and multi-sensitivity of hyperspatial dynamical systems, J. Math. Anal. Appl., 429 (2015), 16-26. 2

[19] X.-X. Wu, X. Wang, G.-R. Chen, On the large deviations of weaker types, Internat. J. Bifur. Chaos Appl. Sci. Engrg., 2017 (2017), 12 pages. 2

[20] X.-X. Wu, L.-D. Wang, G.-R. Chen, Weighted backward shift operators with invariant distributionally scrambled subsets, Ann. Funct. Anal., 8 (2017), 199-210.

[21] X.-X. Wu, L.-D. Wang, J.-H. Liang, The chain properties and average shadowing property of iterated function systems, Qual. Theory Dyn. Syst., 2016 (2016), 9 pages.

[22] J.-C. Xiong, Chaos in a topologically transitive system, Sci. China Ser. A, 48 (2005), 929-939. 1 